\title{
Yield Potential Screening of Desi Chickpea Genotypes in Water Stress Conditions
}

\author{
Alireza Taleei ${ }^{1 *}$ and Jalal Shaabani ${ }^{2}$ \\ ${ }^{1}$ Professor in the Department of Agronomy \& Plant breeding, \\ Faculty of Agricultural Sciences and Engineering, \\ College of Agriculture and Natural Resources, \\ University of Tehran, Karaj, P.O. Box 31587-71787, Iran \\ ${ }^{2}$ Post graduate student in the Department of Agronomy \& Plant breeding, \\ Faculty of Agricultural Sciences and Engineering, \\ College of Agriculture and Natural Resources, \\ University of Tehran, Karaj, P. O. Box: 31587-71787, Iran. \\ lataleei@ut.ac.ir, ${ }^{j}$ shaabani@ut.ac.ir
}

\begin{abstract}
Food legumes are valuable sources of vegetable protein foods, which productivity limited by environmental stresses as drought. To screen some of Iranian drought tolerant Desi chickpea genotypes, twenty-eight lines were assessed with two cultivars namely Pyrooz and Kaka as check varieties and surveying of the drought tolerance indices, too. Cluster analysis on traits-obtained data placed the genotypes 321 and 322 in a same group; these genotypes, moreover, were located with the genotype 10 at a distinc group based on tolerance indices. Total dry matter and grain yield were two characters that have most contribution of first factor; however, second one was justified predominantly by number of seed in the factor analysis. The greatest relationship with yield has shown for total dry matter of this crop. Genotypes with high values for grain yield in stress condition (Ys), stress tolerance index (STI), geometric mean productivity (GMP), and harmonic mean (HARM) were highly drought tolerant ones. In this work genotypes 321, 322, and 10 screened as tolerant ones and those of 407, 261, and 247 were susceptible, respectively. The results showed that the intended genotypes had short time of flowering and podding stages, to apply stress at 50\% flowering time for overall genotypes.
\end{abstract}

Keywords: Cicer ariethinum, drought stress, tolerance indices, factor analysis, cluster analysis

\section{Introduction}

Chickpea as the second most important grain legume crop in the world is cultivated mainly in arid and semi-arid regions. This crop is a main source of protein for many people in the developing countries, chickpea seed, further, has known as a full source of fibre, minerals (phosphorus, calcium, magnesium, iron, and zinc) and $\beta$-carotene that are essential elements in human diet (Singh et al. 2016). Environmental stresses limit growth and productivity of plants, such as drought that has destructive effects on the crops life cycles. To tolerance drought harms and compensate of them, multiple strategies employed by plants. Drought stress causes reduction in leaf area, stem extension, and increase in the root system, also disturbs water relations, and reduces water-use efficiency in crops. On the other hand, plants responses at cellular, as biochemical and/or whole-organs levels to overcome drought stress damages could be very different; hence, making the mechanisms as a very complex phenomenon (Farooq et al. 2009). Most yields of chickpea is ready in marginal regions at the end of spring, and due to lack of rainfall during flowering, 
podding, and seed-filling time, drought is a major abiotic stress, which reduce growth and yield of chickpea in Iran (Sabaghpour et al. 2006). Fang et al. (2010) said that drought stress at flowering time caused devastation in pollen of chickpea and its stigma function. In small grain cereals and legumes, Sadras and Dreccer (2015) found that the critical period to stresses is a little before and after of plant flowering period, respectively.

Improvement and selection of genotypes with higher yield potential and stability under drought stress condition is a great breeding challenge still and has shown low and slow advance and observed in a few crops such as common bean, rice, and maize (White and Castillo, 1992; Fukai and Cooper. 1995; Schneider et al., 1997; Banziger et al., 1999). Ludlow and Muchow (1990) believed that the lack of complete successfully in these aims could be due to quantitative and temporary variability in soil moisture during the evaluating of traits in the experiments between multiple years, and or low genotypic variance in yield of cultivars under drought condition. High complex genetic basis of drought tolerance can be one of those, also (Turner et al. 2001). Lake and Sadras (2016) reported that the associations between yield and crop growth rate were stronger under drought stress than normal conditions in chickpea. Rate of plant growth is dependent on two factors, enviromental and genotypic source of variation, and hence this character is used to modelling and has potential functions in plant breeding (Wiegand and Richardson, 1990). Krishnamurthy et al. (2010) found that heritability of chickpea shoot biomass and seed yield under drought stress were more than those values in normal irrigated condition.

Plant breeders have used many development strategies to increase drought tolerance in crops through time. Selection through tolerance criteria is a one of them, which introduced to segregating genotypes by different responses to drought stress in the field conditions. Hall (1993) reported drought resistance indicates as a relative yield of a genotype subjected to the same drought stress compared to other genotypes. Susceptibility to drought often measured as a function of reduction in yield of a genotype under drought stress, whereas the values are confounded with differential yield potential of genotypes (Ramirez-Vallejo and Kelly, 1998). Multiple selection criteria have given to select tolerant genotypes based on their yields in stress and non-stress conditions. Fischer et al. (1998) proposed relative drought index (RDI), which could be representing genotypes tolerance to drought stress. Drought resistance index (DI) which was introdused by Jusheng (1998), is a confirmed index that used to identify genotypes with higher yield under both stress and without stress conditions. Rosielle and Hamblin (1981) described a stress tolerance index (TOL) as the differences in the amount of yield between drought stress and irrigated environments and mean productivity (MP) as the average yield of genotypes under both stress and non-stress conditions. Severity of drought stress can vary in field environments over years, for this reason, breeders interested in crop relative performance often use the geometric mean productivity (GMP) (Fernandez, 1992). One of the most important tolerance indices is stress tolerance index (STI) that has defined by Fernandez (1992) as a beneficial tool to determine stress tolerance potential of genotypes. For as much as collection a large number of traits, it needed to conserve yield under drought, there is a need to categorize more and more genotypes to introduce diversity in drought tolerance breeding programs (Krishnamurthy et al. 2010).

Accordingly, selection of some Iranian Desi chickpea landraces with higher tolerance to drought stress and screening drought tolerance indices in the field condition, and genotypes with higher yield potential in the average of drought stress and non-stress conditions were objectives of this research.

\section{Materials and Methods}

This research was carried out in the research field of the Department of Agronomy and Plant breeding, University College of Agriculture and Natural Resources, University of Tehran-Karaj, Iran (with latitude $35^{\circ} 56^{\prime} \mathrm{N}$ and longitude $50^{\circ} 58^{\prime} \mathrm{E}$ and altitude of $1112.5 \mathrm{~m}$ 
above sea level) between February and August 2014. The average annual rainfall based on data of 48 years average is $268 \mathrm{~mm}$ and the amount of rainfall for the research period was $94.5 \mathrm{~mm}$.

\subsection{Plant Materials and Experimental Design}

Twenty-eight Desi chickpea lines selected from departmental gene bank along with two cultivars namely Pyrooz and Kaka as controls shown in Table 1. A nested completely randomized block design with two replications used to implement the experiment. Each block considered as an environment and all genotypes randomly allocated in each block, in a way that two environments contain drought stress and non-stress conditions. The seeds of each line sown in rows with 1-meter length and between rows distance of $0.5 \mathrm{~m}$, and that of between plants were $10 \mathrm{~cm}$. The experiment consists of four blocks, two for drought, and two for non-stress conditions.

\subsection{Data Collection}

Days to $50 \%$ flowering ( $\mathrm{Fl}$ ), 50\% podding (Po) and 50\% maturity (Ma), as phenological traits recorded for every rows during developmental stage of plants. Considering the marginal effects, equal number of plants for each line harvested. The rest of traits measured after harvesting of plants including yield (Yi), total dry matter (TDM), 100 -seed weight $(\mathrm{Sw})$, and harvest index $(\mathrm{HI})$. An electronic weighing scale used to measure those. In addition, number of seeds (Ns) also recorded. Drought stress applied in $50 \%$ of flowering time for all blocks and since then irrigation terminated in stress condition, however, in non-stress condition continued and it was due to common irrigation regime of the region.

The tolerance indices such as mean productivity (MP), tolerance index (TOL), geometric mean productivity (GMP), stress tolerance index (STI), harmonic mean (HARM), relative drought index (RDI), and drought resistance index (DI) obtained using the following formulas:

$$
\begin{aligned}
& \mathrm{MP}=(\mathrm{Yp}+\mathrm{Ys}) / 2 \\
& \mathrm{TOL}=\mathrm{Yp}-\mathrm{Ys} \\
& \mathrm{GMP}=\sqrt{\mathrm{Yp}+\mathrm{YS}_{S}} \\
& \mathrm{STI}=(\mathrm{Yp} \times \mathrm{Ys}) /(\mathrm{Yp})^{2} \\
& \mathrm{HARM}=[2(\mathrm{Yp} \times \mathrm{Ys})] /(\mathrm{Yp}+\mathrm{Ys}) \\
& \mathrm{RDI}=(\mathrm{Ys} / \mathrm{Yp}) /\left(\overline{\mathrm{Y}}_{\mathrm{S}} / \overline{\mathrm{Y}}_{\mathrm{p}}\right) \\
& \mathrm{DI}=[\mathrm{Ys} \times(\mathrm{Ys} / \mathrm{Yp})] / \overline{\mathrm{Y}}_{\mathrm{S}}
\end{aligned}
$$

Where, Ys and Yp represent yield in stress and non-stress conditions, respectively, while $\overline{\mathrm{Y}}_{\mathrm{S}}$ and $\overline{\mathrm{Y}}_{\mathrm{p}}$ are mean yield of all genotypes in stress and non-stress conditions, too.

\subsection{Data Analysis}

To eliminate the probable errors, the average of two replications was used to analysis. One-way analysis of variance applied for scored traits. Besides, the above-mentioned indices calculated for stress and non-stress conditions, respectively. The genotype's mean yield of each environment compared. In addition, multivariate analyses used for the traits and tolerance indices. The obtained data subjected to analysis of variance (ANOVA) with the Statistical Software Package (SAS, version 9.3, SAS Institute Inc. Cary, NC, USA). Factor analysis, principal component analysis, cluster analysis, and biplot analysis as multivariate techniques performed using Statgraphics X64 (Statgraphics Centurion XV1.11, Stat Point Technologies, USA). 


\section{Table 1. The Line Number and Origin of Evaluated Desi Chickpea Genotypes for Water Stress Conditions}

\begin{tabular}{cccc}
\hline Lines No. & Origin & Lines No. & Origin \\
\hline 5 & Iran & 151 & Iran \\
8 & Iran & 231 & Iran \\
9 & Iran & 232 & Iran \\
10 & Iran & 247 & Iran \\
21 & Iran & 252 & Iran \\
46 & Iran & 267 & Iran \\
47 & Iran & 276 & Iran \\
48 & Iran & 316 & Iran \\
49 & Iran & 321 & Iran \\
50 & Iran & 322 & Iran \\
51 & Iran & 333 & Iran \\
76 & Iran & 347 & Iran \\
90 & Iran & 407 & Iran \\
122 & Iran & Pyrooz & Iran \\
150 & Iran & Kaka & Iran \\
\hline & & &
\end{tabular}

\section{Results}

\subsection{Phenological Traits}

Flowering time variance was not significant among genotypes, yet its range varied from $62.50 \pm 3.31$ to $77.50 \pm 10.23$ days for genotypes 267 and 276, respectively. According to results for podding time, drought stress has significant effect on the character $(P \leq$ $0.01)$, as genotype $267(70.00 \pm 1.73)$ and genotype $276(81.00 \pm 6.50)$ have lowest and highest times to rise of pod. High significant effect of drought stress on times to maturity was observed $(P \leq 0.01)$ and minimum of days to maturity was seen in genotype 252 (85.00 \pm 31.21 ), also the maximum of time to maturity was belonged to genotype 8 by $110.0 \pm 12.32$ days (Table 2).

\subsection{Total Dry Matter and Harvest Index}

The results of ANOVA in Table 2 showed that both of the total dry matter and harvest index affected by drought stress, which were highly significant. Among the evaluated genotypes, genotype 5 with $23.04 \pm 9.48$ gr plant $^{-1}$ and genotype 407 with $6.26 \pm 5.32$ gr plant $^{-1}$ have highest and lowest total dry matter, respectively. Genotype 333 has the highest harvest index $(0.65 \pm 0.24)$, while that of genotype $5(0.32 \pm 0.04)$ was the lowest one (Table 2).

\subsection{Yield and Its Components}

The tested genotypes have not affected by drought stress for 100-seed weight; however, the character showed highly significant difference between genotypes within environments $(P \leq 0.01)$. According to Table 2 , grain yield and seed number showed highly significant differences in stress and non-stress conditions. Results of means comparisons, have been shown in Table 2, revealed that $3.68 \pm 2.49$ gr plant $^{-1}$ and 
$10.48 \pm 4.85$ gr plant $^{-1}$ were lowest and highest grain yield which belonged to genotypes 407 and 321, respectively. The genotype 407 with $22.39 \pm 12.37$ seeds plant ${ }^{-1}$ has the minimum and the genotype 321 with $69.01 \pm 31.54$ has the maximum of seed number per plant. The genotypes 407 and $321(10.07 \pm 0.14)$ and the genotype $347(40.66 \pm 21.74)$ showed most and least 100-seed weight per plant (Table 2).

Table 2. Comparison of Trait Means for Genotypes per Single Plant Level in Stress and Non-Stress Conditions

\begin{tabular}{|c|c|c|c|c|c|c|c|c|}
\hline \multicolumn{9}{|c|}{ Traits } \\
\hline Genotype & $\begin{array}{l}\text { FL } \\
\text { (day) }\end{array}$ & $\begin{array}{c}\mathrm{PO} \\
\text { (day) }\end{array}$ & $\begin{array}{l}\text { MA } \\
\text { (day) }\end{array}$ & $\begin{array}{c}\text { TDM } \\
\left(\text { gr plant }^{-1}\right)\end{array}$ & $\begin{array}{c}\text { NS } \\
\left(\text { Plant }^{-1}\right)\end{array}$ & $\begin{array}{c}\text { YI } \\
\left(\text { gr plant }^{-1}\right)\end{array}$ & $\begin{array}{l}\text { SW } \\
\text { (gr) }\end{array}$ & $\begin{array}{c}\mathrm{HI} \\
(\%)\end{array}$ \\
\hline Pyrooz & $67.5 \pm 7.22$ & $74.5 \pm 4.35$ & $96.5 \pm 4.78$ & $12.74 \pm 6.29$ & $36.13 \pm 19.17$ & $5.26 \pm 2.64$ & $14.90 \pm 0.61$ & $0.41 \pm 0.02$ \\
\hline Kaka & $66.0 \pm 5.50$ & $72.5 \pm 1.82$ & $102.0 \pm 8.04$ & $9.41 \pm 4.30$ & $40.45 \pm 16.52$ & $4.89 \pm 2.16$ & $11.90 \pm 1.17$ & $0.52 \pm 0.07$ \\
\hline 5 & $66.5 \pm 3.59$ & $71.0 \pm 3.77$ & $102.5 \pm 6.35$ & $23.04 \pm 9.48$ & $33.92 \pm 15.88$ & $7.54 \pm 3.49$ & $22.15 \pm 1.92$ & $0.32 \pm 0.04$ \\
\hline 8 & $65.5 \pm 2.21$ & $73.0 \pm 2.82$ & $110.0 \pm 12.32$ & $17.20 \pm 4.96$ & $27.51 \pm 9.371$ & $7.55 \pm 3.04$ & $27.74 \pm 6.20$ & $0.42 \pm 0.06$ \\
\hline 9 & $63.5 \pm 2.21$ & $74.0 \pm 1.82$ & $103.5 \pm 5.94$ & $17.51 \pm 7.65$ & $29.46 \pm 17.55$ & $7.39 \pm 4.74$ & $24.12 \pm 2.04$ & $0.38 \pm 0.11$ \\
\hline 10 & $66.5 \pm 5.18$ & $72.0 \pm 04.76$ & $99.0 \pm 03.09$ & $19.44 \pm 4.37$ & $42.51 \pm 12.63$ & $8.72 \pm 3.02$ & $20.33 \pm 2.71$ & $0.44 \pm 0.09$ \\
\hline 21 & $72.5 \pm 2.50$ & $71.5 \pm 1.41$ & $101.5 \pm 6.75$ & $12.53 \pm 2.43$ & $38.65 \pm 9.83$ & $5.96 \pm 1.69$ & $15.24 \pm 0.76$ & $0.46 \pm 0.04$ \\
\hline 46 & $72.5 \pm 4.79$ & $76.0 \pm 2.06$ & $101.5 \pm 11.04$ & $13.37 \pm 3.86$ & $55.37 \pm 19.16$ & $5.62 \pm 1.77$ & $10.45 \pm 1.31$ & $0.42 \pm 0.04$ \\
\hline 47 & $69.0 \pm 4.03$ & $74.5 \pm 2.44$ & $102.0 \pm 4.78$ & $13.30 \pm 3.99$ & $62.75 \pm 17.22$ & $6.31 \pm 1.72$ & $10.07 \pm 0.14$ & $0.48 \pm 0.05$ \\
\hline 48 & $64.0 \pm 10.59$ & $71.5 \pm 10.09$ & $102.0 \pm 11.26$ & $12.73 \pm 6.70$ & $53.77 \pm 30.27$ & $6.27 \pm 3.16$ & $11.62 \pm 0.84$ & $0.48 \pm 0.03$ \\
\hline 49 & $68.5 \pm 3.74$ & $73.5 \pm 2.38$ & $101.0 \pm 6.70$ & $13.40 \pm 12.98$ & $60.20 \pm 29.22$ & $6.39 \pm 3.21$ & $10.58 \pm 0.54$ & $0.46 \pm 0.13$ \\
\hline 50 & $63.5 \pm 14.31$ & $72.0 \pm 11.70$ & $97.5 \pm 12.98$ & $13.74 \pm 4.49$ & $60.97 \pm 21.50$ & $6.86 \pm 2.67$ & $10.95 \pm 1.02$ & $0.48 \pm 0.03$ \\
\hline 51 & $68.5 \pm 7.14$ & $74.0 \pm 4.24$ & $101.5 \pm 6.85$ & $11.29 \pm 2.60$ & $47.64 \pm 13.00$ & $5.26 \pm 1.43$ & $11.16 \pm 0.88$ & $0.46 \pm 0.06$ \\
\hline 76 & $69.5 \pm 6.73$ & $80.0 \pm 7.88$ & $107.75 \pm 13.32$ & $9.52 \pm 3.06$ & $37.56 \pm 11.26$ & $4.12 \pm 1.29$ & $10.94 \pm 1.58$ & $0.43 \pm 0.04$ \\
\hline 90 & $68.5 \pm 6.80$ & $74.0 \pm 3.00$ & $100.5 \pm 11.11$ & $14.51 \pm 4.06$ & $63.86 \pm 19.25$ & $7.31 \pm 2.13$ & $11.59 \pm 0.56$ & $0.50 \pm 0.02$ \\
\hline 122 & $71.5 \pm 10.2$ & $78.0 \pm 9.14$ & $106.5 \pm 17.79$ & $13.20 \pm 3.82$ & $52.84 \pm 16.38$ & $5.70 \pm 1.58$ & $10.91 \pm 0.74$ & $0.43 \pm 0.03$ \\
\hline 150 & $67.5 \pm 5.50$ & $74.5 \pm 2.21$ & $100.0 \pm 4.04$ & $11.05 \pm 2.79$ & $38.02 \pm 6.65$ & $4.56 \pm 1.04$ & $12.10 \pm 0.97$ & $0.42 \pm 0.04$ \\
\hline 151 & $67.0 \pm 5.19$ & $76.0 \pm 5.65$ & $100.5 \pm 21.88$ & $14.99 \pm 6.96$ & $51.04 \pm 28.28$ & $5.72 \pm 2.72$ & $12.41 \pm 2.69$ & $0.40 \pm 0.08$ \\
\hline 231 & $70.0 \pm 2.16$ & $76.5 \pm 0.50$ & $88.5 \pm 17.72$ & $10.86 \pm 3.48$ & $30.17 \pm 12.80$ & $4.93 \pm 2.81$ & $16.34 \pm 3.19$ & $0.44 \pm 0.13$ \\
\hline 232 & $69.0 \pm 13.52$ & $73.0 \pm 12.57$ & $100.5 \pm 10.23$ & $12.97 \pm 2.89$ & $39.87 \pm 15.18$ & $5.98 \pm 2.87$ & $15.02 \pm 3.11$ & $0.46 \pm 0.18$ \\
\hline 247 & $63.0 \pm 6.29$ & $71.5 \pm 4.19$ & $96.0 \pm 4.85$ & $9.22 \pm 3.05$ & $42.11 \pm 12.27$ & $4.39 \pm 1.40$ & $10.29 \pm 0.55$ & $0.47 \pm 0.04$ \\
\hline 252 & $71.0 \pm 0.50$ & $75.5 \pm 1.70$ & $85.0 \pm 31.21$ & $11.26 \pm 2.40$ & $31.06 \pm 4.73$ & $4.43 \pm 0.84$ & $14.30 \pm 0.94$ & $0.39 \pm 0.06$ \\
\hline 267 & $62.5 \pm 3.31$ & $70.0 \pm 1.73$ & $98.5 \pm 5.85$ & $9.31 \pm 2.74$ & $42.04 \pm 13.07$ & $4.78 \pm 1.70$ & $11.47 \pm 1.67$ & $0.51 \pm 0.07$ \\
\hline 276 & $77.5 \pm 10.23$ & $81.0 \pm 6.50$ & $101.0 \pm 5.71$ & $13.00 \pm 5.25$ & $55.70 \pm 24.74$ & $8.80 \pm 5.21$ & $14.85 \pm 3.82$ & $0.62 \pm 0.18$ \\
\hline 316 & $65.5 \pm 2.87$ & $71.0 \pm 1.70$ & $101.5 \pm 7.50$ & $11.47 \pm 3.70$ & $31.79 \pm 14.86$ & $5.16 \pm 2.66$ & $16.22 \pm 4.02$ & $0.44 \pm 0.17$ \\
\hline 321 & $63.0 \pm 3.78$ & $71.5 \pm 1.73$ & $100.5 \pm 7.70$ & $21.06 \pm 8.64$ & $69.01 \pm 31.54$ & $10.48 \pm 4.85$ & $15.45 \pm 4.39$ & $0.49 \pm 0.25$ \\
\hline 322 & $64.5 \pm 2.62$ & $75.0 \pm 1.00$ & $101.0 \pm 6.18$ & $19.14 \pm 9.78$ & $61.74 \pm 36.09$ & $9.55 \pm 6.05$ & $16.02 \pm 1.66$ & $0.50 \pm 0.08$ \\
\hline 333 & $64.0 \pm 3.55$ & $73.0 \pm 2.38$ & $100.5 \pm 3.77$ & $7.95 \pm 5.91$ & $35.05 \pm 18.69$ & $5.50 \pm 3.01$ & $15.52 \pm 0.61$ & $0.65 \pm 0.24$ \\
\hline 347 & $68.0 \pm 2.64$ & $74.25 \pm 2.08$ & $100.5 \pm 4.76$ & $18.50 \pm 7.58$ & $34.35 \pm 21.53$ & $5.61 \pm 2.05$ & $40.66 \pm 21.74$ & $0.32 \pm 0.06$ \\
\hline 407 & $66.5 \pm 13.67$ & $70.5 \pm 2.44$ & $95.5 \pm 7.41$ & $6.26 \pm 5.32$ & $22.39 \pm 12.37$ & $3.68 \pm 2.49$ & $16.32 \pm 1.09$ & $0.59 \pm 0.17$ \\
\hline $\begin{array}{c}\text { Environme } \\
\text { nt }\end{array}$ & $* *$ & $* *$ & $* *$ & $* *$ & $* *$ & $* *$ & ns & $* *$ \\
\hline $\begin{array}{c}\text { Genotype } \\
\text { /Environme } \\
\text { nt }\end{array}$ & ns & ns & ns & ns & ns & ns & $* *$ & ns \\
\hline $\mathrm{LSD}_{0.05}$ & 2.18 & 1.66 & 2.99 & 1.92 & 6.22 & 0.82 & 4.17 & 0.03 \\
\hline $\mathrm{CV}(\%)$ & 2.23 & 1.41 & 1.92 & 15.04 & 13.89 & 34.98 & 9.45 & 13.25 \\
\hline
\end{tabular}

Each value is the mean of two replicates \pm standard deviation $(\mathrm{Sd})$. Fisher protected LSD at $\mathrm{P} \leq 0.05$. FL: days to $50 \%$ of flowering, PO: days to $50 \%$ of podding, MA: days to $50 \%$ of maturity, TDM: total dry matter, YI: grain yield, NS: number of seed, SW: 100seed weight, HI: harvest index.

**: significant at $1 \%$ level, ns: non-significant, CV: Coefficient of Variation. 


\subsection{Tolerance Indices}

The highest values of Ys, MP, DI, GMP, STI, and HARM indices observed for genotype 321, while those of Yp and TOL have seen for genotype 276; genotype 252, also, has highest RDI value index. However, the fewest values of MP, GMP, STI, and HARM indices were belonged to genotype 407. The genotype 9, in addition, has the fewest amounts of DI and RDI. The low values of Ys and TOL observed in Pyrooz and genotype 252 , respectively (Table 3 ).

\section{Table 3. Calculated Indices of Drought Tolerance for Genotypes per Single} Plant

\begin{tabular}{|c|c|c|c|c|c|c|c|c|c|}
\hline $\begin{array}{c}\text { Genotype } \\
\text { No. }\end{array}$ & Yp & Ys & MP & TOL & GMP & STI & HARM & RDI & DI \\
\hline Pyrooz & 8.000 & 2.525 & 5.262 & 5.475 & 4.494 & 0.321 & 3.838 & 0.565 & 0.180 \\
\hline Kaka & 6.578 & 3.208 & 4.893 & 3.370 & 4.594 & 0.336 & 4.313 & 0.873 & 0.353 \\
\hline 5 & 10.714 & 4.384 & 7.549 & 6.329 & 6.854 & 0.748 & 6.222 & 0.732 & 0.405 \\
\hline 8 & 10.636 & 4.476 & 7.556 & 6.160 & 6.900 & 0.758 & 6.300 & 0.753 & 0.425 \\
\hline 9 & 11.923 & 2.863 & 7.393 & 9.059 & 5.843 & 0.543 & 4.618 & 0.430 & 0.155 \\
\hline 10 & 10.590 & 6.857 & 8.724 & 3.733 & 8.521 & 1.156 & 8.324 & 1.159 & 1.003 \\
\hline 21 & 7.461 & 4.461 & 5.961 & 3.000 & 5.769 & 0.530 & 5.584 & 1.070 & 0.602 \\
\hline 46 & 5.875 & 5.375 & 5.625 & 0.500 & 5.619 & 0.503 & 5.613 & 1.638 & 1.111 \\
\hline 47 & 6.777 & 5.857 & 6.317 & 0.920 & 6.300 & 0.632 & 6.283 & 1.547 & 1.144 \\
\hline 48 & 9.000 & 3.555 & 6.277 & 5.444 & 5.656 & 0.509 & 5.097 & 0.707 & 0.317 \\
\hline 49 & 9.258 & 3.500 & 6.392 & 5.785 & 5.700 & 0.517 & 5.083 & 0.674 & 0.298 \\
\hline 50 & 9.647 & 4.081 & 6.864 & 5.565 & 6.275 & 0.627 & 5.736 & 0.757 & 0.390 \\
\hline 51 & 5.695 & 4.833 & 5.264 & 0.862 & 5.246 & 0.438 & 5.229 & 1.519 & 0.927 \\
\hline 76 & 4.633 & 3.622 & 4.128 & 1.010 & 4.096 & 0.267 & 4.066 & 1.400 & 0.640 \\
\hline 90 & 9.200 & 5.437 & 7.318 & 3.762 & 7.072 & 0.796 & 6.835 & 1.058 & 0.726 \\
\hline 122 & 6.071 & 5.333 & 5.702 & 0.738 & 5.690 & 0.515 & 5.678 & 1.573 & 1.058 \\
\hline 150 & 5.181 & 3.952 & 4.567 & 1.229 & 4.525 & 0.326 & 4.484 & 1.365 & 0.681 \\
\hline 151 & 6.571 & 4.875 & 5.723 & 1.696 & 5.660 & 0.510 & 5.597 & 1.328 & 0.817 \\
\hline 231 & 6.722 & 3.142 & 4.932 & 3.579 & 4.596 & 0.336 & 4.283 & 0.837 & 0.332 \\
\hline 232 & 6.575 & 5.391 & 5.983 & 1.183 & 5.953 & 0.564 & 5.924 & 1.468 & 0.999 \\
\hline 247 & 5.650 & 3.146 & 4.398 & 2.503 & 4.216 & 0.283 & 4.041 & 0.997 & 0.396 \\
\hline 252 & 4.600 & 4.266 & 4.433 & 0.333 & 4.430 & 0.312 & 4.427 & 1.661 & 0.894 \\
\hline 267 & 5.894 & 3.678 & 4.786 & 2.216 & 4.656 & 0.345 & 4.530 & 1.117 & 0.518 \\
\hline 276 & 13.600 & 4.000 & 8.800 & 9.600 & 7.375 & 0.866 & 6.181 & 0.526 & 0.265 \\
\hline 316 & 6.612 & 4.400 & 5.506 & 2.212 & 5.394 & 0.463 & 5.284 & 1.191 & 0.661 \\
\hline 321 & 11.888 & 9.083 & 10.486 & 2.805 & 10.391 & 1.720 & 10.298 & 1.368 & 1.568 \\
\hline 322 & 13.187 & 5.923 & 9.555 & 7.264 & 8.838 & 1.244 & 8.174 & 0.804 & 0.601 \\
\hline 333 & 8.142 & 2.875 & 5.508 & 5.267 & 4.838 & 0.372 & 4.249 & 0.632 & 0.229 \\
\hline 347 & 6.400 & 4.834 & 5.617 & 1.565 & 5.562 & 0.492 & 5.508 & 1.352 & 0.825 \\
\hline 407 & 4.575 & 2.790 & 3.682 & 1.784 & 3.573 & 0.203 & 3.466 & 1.092 & 0.384 \\
\hline
\end{tabular}

Where, Yp: grain yield in non-stress condition, Ys: grain yield in stress condition, MP: mean productivity, TOL: tolerance index, GMP: geometric mean productivity, STI: stress tolerance index, HARM: harmonic mean, RDI: relative drought index, DI: drought resistance index.

\subsection{Partial Correlations}

\subsubsection{Non-Stress Condition}

The correlation of flowering and podding time, among phenological of Desi chickpea traits, also podding and maturity of the crop were highly significant. Total dry matter and harvest index did not show any relationship with the traits related to phenology and maturity; however, those correlation with grain yield was significant and highly significant, respectively. Seed number and 100 -seed weight, have highly negative correlation, nevertheless, there were positive and highly significant correlations between grain yield and both of them. Harvest index and time of podding, in contrary to maturity of the plant, showed a positive significant correlation with yield (Table 4). 


\subsubsection{Stress Condition}

Only two characters have a correlation and of cource severely positive with yield. On the other hand, it has a negative relationship between those, total dry matter and harvest index. The correlation between number of seed and 100-seed weight was significant and negative, too (Table 5).

\subsubsection{Stress and Non-Stress Conditions' Mean}

The phenological measured traits, shown in Table 6, did not have any relationships to that of yield and its components. Total dry matter and harvest index have highly, as 100seed weight, which has a significant positive correlation with grain yield. A high negative correlation, in addition, found between total dry matter with harvest index and between 100 -seed weight and seed number, too.

Table 4. Partial Correlation Coefficients between Traits at Non-Stress Condition

\begin{tabular}{cccccccc}
\hline & Flowering & Podding & Maturity & Total dry matter & Seed No. & Yield & 100 -seed weight \\
\hline Podding & $0.771^{* *}$ & & & & & \\
Maturity & $-0.193^{\text {ns }}$ & $0.519^{* *}$ & & & & \\
Total dry matter & $-0.123^{\text {ns }}$ & $-0.051^{\text {ns }}$ & $-0.080^{\text {ns }}$ & & & \\
Seed No. & $0.181^{\text {ns }}$ & $-0.508^{*}$ & $0.524^{* *}$ & $-0.008^{\text {ns }}$ & & \\
Yield & $-0.133^{\text {ns }}$ & $0.504^{*}$ & $-0.459^{*}$ & $0.404^{*}$ & $0.905^{* *}$ & & \\
100 -seed weight & $0.209^{\text {ns }}$ & $-0.565^{* *}$ & $0.600^{* *}$ & $0.039^{\text {ns }}$ & $-0.977^{* *}$ & $0.882^{* *}$ & $-0.015^{\text {ns }}$ \\
Haevest index & $-0.103^{\text {ns }}$ & $-0.068^{\text {ns }}$ & $-0.079^{\text {ns }}$ & $-0.916^{* *}$ & $-0.069^{\text {ns }}$ & $0.434^{*}$ & \\
\hline
\end{tabular}

**: significant at $1 \%$ level, *: significant at $5 \%$ level, ns: non-significant

Table 5. Partial Correlation Coefficients between Traits at Stress Condition

\begin{tabular}{|c|c|c|c|c|c|c|c|}
\hline & Flowering & Podding & Maturity & Total dry matter & Seed No. & Yield & 100-seed weight \\
\hline Podding & $0.774^{* *}$ & & & & & & \\
\hline Maturity & $-0.258^{\mathrm{ns}}$ & $0.120^{\mathrm{ns}}$ & & & & & \\
\hline Total dry matter & $0.026^{\mathrm{ns}}$ & $0.022^{\text {ns }}$ & $0.265^{\mathrm{ns}}$ & & & & \\
\hline Seed No. & $0.059^{\mathrm{ns}}$ & $0.031^{\mathrm{ns}}$ & $0.121^{\mathrm{ns}}$ & $-0.132^{\mathrm{ns}}$ & & & \\
\hline Yield & $-0.001^{\mathrm{ns}}$ & $-0.051^{\mathrm{ns}}$ & $-0.243^{\mathrm{ns}}$ & $0.977^{* *}$ & $0.292^{\mathrm{ns}}$ & & \\
\hline 100 -seed weight & $-0.055^{\mathrm{ns}}$ & $0.178^{\mathrm{ns}}$ & $0.182^{\text {ns }}$ & $-0.100^{\mathrm{ns}}$ & $-0.461^{*}$ & $0.199^{\text {ns }}$ & \\
\hline Haevest index & $-0.020^{\mathrm{ns}}$ & $0.015^{\mathrm{ns}}$ & $0.276^{\mathrm{ns}}$ & $-0.933^{* *}$ & $0.084^{\mathrm{ns}}$ & $0.890^{* * *}$ & $-0.121^{\mathrm{ns}}$ \\
\hline
\end{tabular}

Table 6. Partial Mean Correlation Coefficients between Traits at Average of Stress and Non-Stress Conditions

\begin{tabular}{|c|c|c|c|c|c|c|c|}
\hline & Flowering & Podding & Maturity & $\begin{array}{c}\text { Total dry } \\
\text { matter }\end{array}$ & Seed No. & Yield & $\begin{array}{c}\text { 100-seed } \\
\text { weight }\end{array}$ \\
\hline Podding & $0.794^{* *}$ & & & & & & \\
\hline Maturity & $-0.151^{\mathrm{ns}}$ & $0.192^{\mathrm{ns}}$ & & & & & \\
\hline Total dry matter & $0.034^{\mathrm{ns}}$ & $-0.246^{\mathrm{ns}}$ & $0.103^{\mathrm{ns}}$ & & & & \\
\hline Seed No. & $-0.025^{\mathrm{ns}}$ & $0.138^{\text {ns }}$ & $0.074^{\mathrm{ns}}$ & $0.198^{\mathrm{ns}}$ & & & \\
\hline Yield & $-0.057^{\mathrm{ns}}$ & $0.233^{\text {ns }}$ & $-0.066^{\mathrm{ns}}$ & $0.941^{* *}$ & $0.052^{\mathrm{ns}}$ & & \\
\hline 100 -seed weight & $0.026^{\mathrm{ns}}$ & $0.075^{\mathrm{ns}}$ & $0.056^{\mathrm{ns}}$ & $0.461^{*}$ & $-0.686^{* *}$ & $-0.247^{\mathrm{ns}}$ & \\
\hline Haevest index & $0.031^{\mathrm{ns}}$ & $-0.240^{\mathrm{ns}}$ & $0.093^{\mathrm{ns}}$ & $-0.932^{* *}$ & $0.138^{\mathrm{ns}}$ & $0.904^{* *}$ & $0.294^{\mathrm{ns}}$ \\
\hline
\end{tabular}

\subsection{Cluster Analysis}

\subsubsection{Characters}

Cluster analysis results using Ward's method shown in Figure 1 for genotypes. According to these results, four groups obtained. The first group included genotypes 276, 231, 76, 151, 46, 252, 232, 51, 150, 122, and Pyrooz. The genotypes 21, 247, 407, 333, 267,316 , and Kaka were placed in the second group. The third group included genotypes 
$47,49,48,90,50,321$, and 322 , as well the genotypes $5,347,10,9$, and 8 included in groupe 4.

\subsubsection{Indices}

The Ward's method devided the genotypes to three groups through their tolerance index values. According to this analysis, the first group included ten genotypes namely Pyrooz, 333, 48, 49, 50, 5, 8, 90, 276, 322, 321. The genotypes 21, 316, 151, 347, 46, 122 , 51,47 , and 232 were identified in the second group and the third group, consisted of genotypes 76, 150, 252, 231, Kaka, 247, 267, and 407 (Figure2).

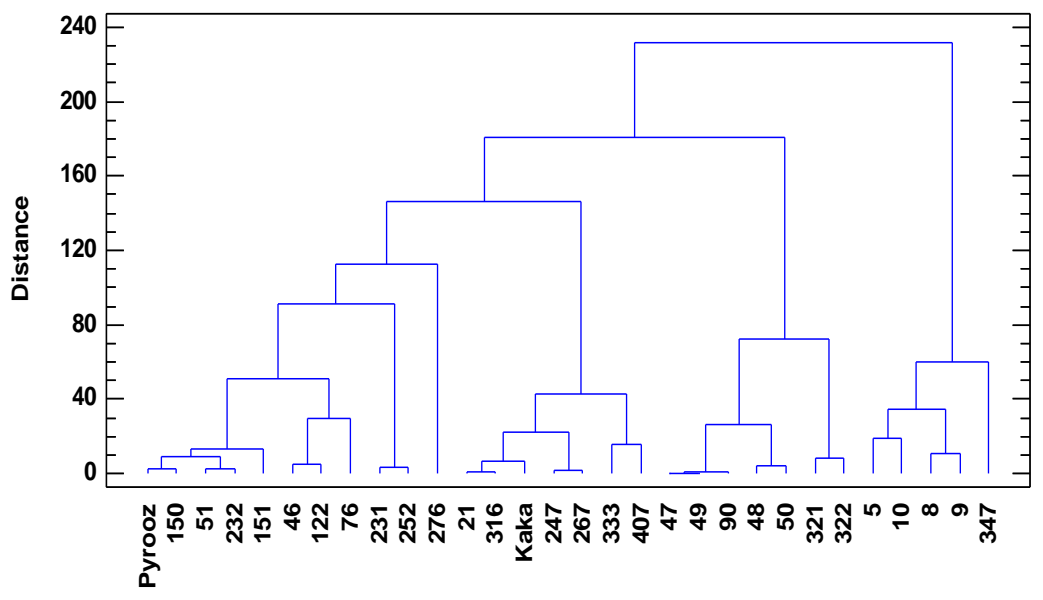

Figure 1. Dendrogram of Mean Genotypes Using Stress and Non-Stress Conditions

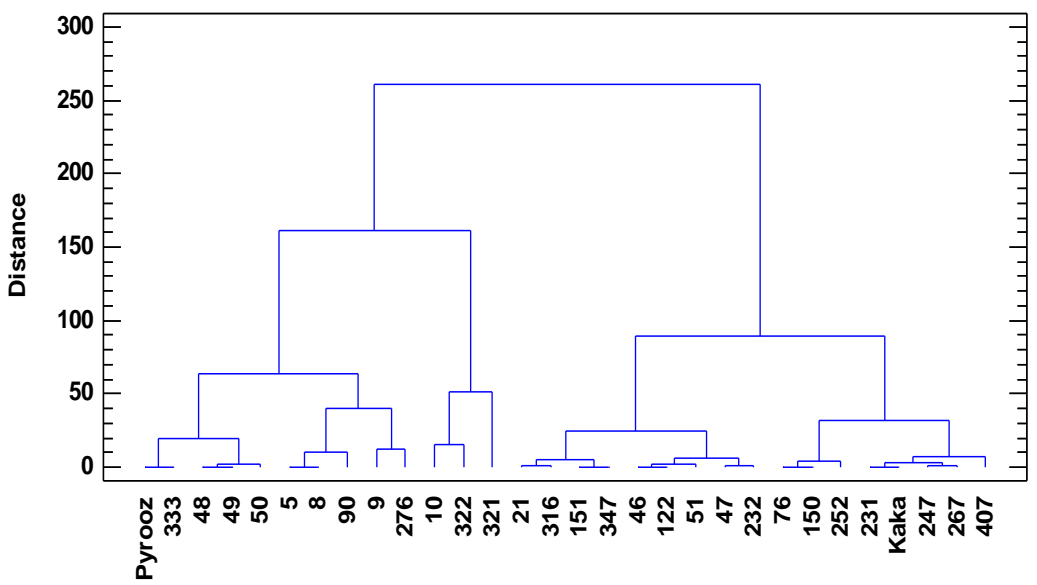

Figure 2. Dendrogram of Genotypes for Indices

\subsection{Factor Analysis}

Factor analysis carried out using principal component method. In this analysis, three factors obtained which justified $76.14 \%$ of total variance. Total dry matter and grain yield have high values for the first factor, which justified $30.38 \%$ of total variation. Number of seeds explained second factor with $24.43 \%$ of variance proportion. The third factor, which explained $21.32 \%$ of total variation, has high value for the flowering time (Tables 7 and 8). 
Table 7. Eigen Values for the Mean Correlation Matrix of Traits Scored in the Stress and Non-Stress Conditions

\begin{tabular}{ccccc}
\hline Factor & Eigen value & Difference & Proportion of variance & Cumulative (\%) \\
\hline 1 & 2.430 & 0.476 & 0.303 & 30.38 \\
2 & 1.954 & 0.248 & 0.244 & 54.81 \\
3 & 1.706 & & 0.213 & 76.14 \\
\hline
\end{tabular}

Table 8. Mean Components Matrix for Traits Using Stress and Non-Stress Conditions

\begin{tabular}{lccl}
\hline Traits & Factor 1 & Factor 2 & Factor 3 \\
\hline Flowering & -0.137 & 0.418 & $\mathbf{0 . 5 4 4}$ \\
Podding & -0.090 & 0.495 & 0.487 \\
Maturity & 0.276 & 0.177 & -0.019 \\
Total dry matter & $\mathbf{0 . 6 2 0}$ & 0.034 & 0.062 \\
Seed No. & 0.167 & $\mathbf{0 . 5 3 1}$ & -0.348 \\
Yield & $\mathbf{0 . 5 1 5}$ & 0.278 & -0.200 \\
100-seed weight & 0.371 & -0.348 & 0.341 \\
Harvest index & -0.281 & 0.253 & -0.427 \\
\hline
\end{tabular}

\subsection{Biplot Analysis}

\subsubsection{Characters}

Figure 3 shows a PCA analysis-mediated 3-D plot, where in the genotypes 321, 322, 10,5 , and 8 were determined as premier genotypes than other; althogh their total dry matter, seed number, and grain yield were higher. A same direct releavance manifested for time of plant maturity and total dry matter.

\subsubsection{Indices}

Biplot analysis based on drought tolerance indices of the Desi chickpea genotypes releaved that GMP and STI have a sever relationship together and a relative strong with HARM index. Yp and Ys, on the other hand, have not a correlation together and each of those indices. The genotypes 276, 9, 8, and 5 have high TOL value; meantime the index has a negative link with RDI, for genotypes 46, 232, and 122. Considering the plot showed that the most tolerant genotypes were 10 and 321, but yield of those was not very favourable at non-stress condition. The high value for grain yield has seen for genotype 322 , where the highest values of Yp and MP also obtained. 


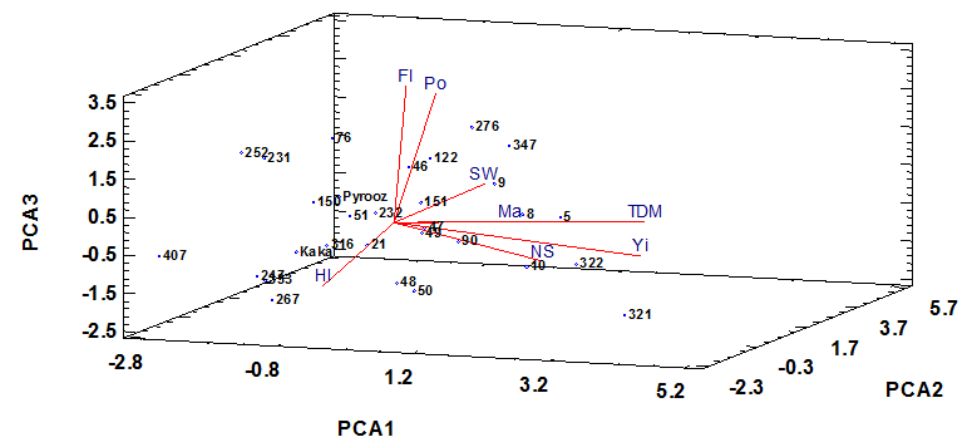

Figure 3. 3-D Plot Analysis for Traits Using PCA 1, 2, and 3

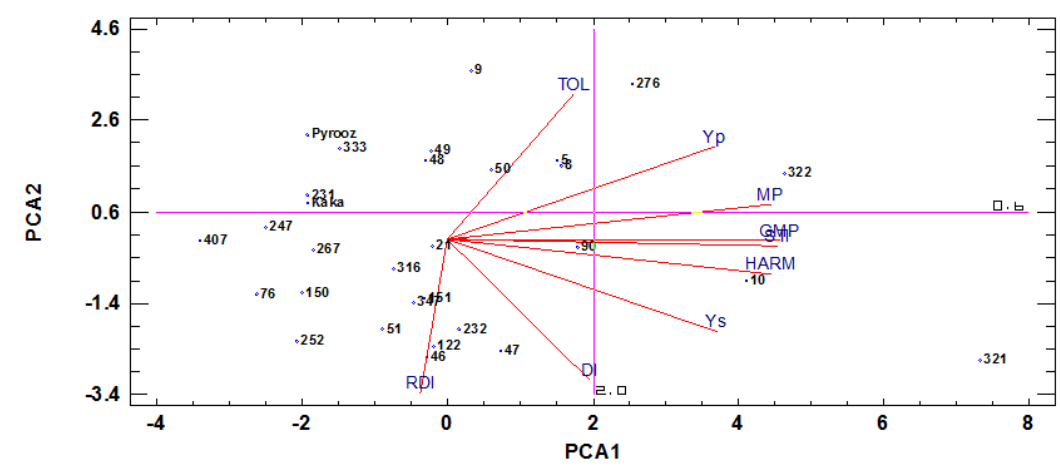

Figure 4. Biplot Analysis for Indices Using PCA 1 and 2

\section{Discussion}

The existence of a large variation has established by this work for drought response in the evaluating set of Desi chickpea genotypes in the field. This collection was included Iranian landraces that adapted to the region during years. The phenological traits, it was seen that have not a main-direct influence on related characters to yield. Because of applying stress was in the $50 \%$ of flowering, this can one reason of the phenomen. However, drought stress impressed these traits and a significant drought-induced difference has shown between stress and non-stress conditions. Nevertheless, maturity time showed a same direction with total dry matter (Figure 3); based on this result, due to exist a possitive correlation between yield and total dry matter, could be said, probably, enhancement in maturity time caused more growth and followed by increase in available period for material remobilization can be resulted to more production in the plant. In other words, more staying immature phases consequently, more photosynthetically activities and production. There has said that the delay in maturity can provide a vital period to hold $\mathrm{CO} 2$ assimilation for photosynthesis (Farooq et al. 2016). Moreover, there has not fonud an association between shorter growth duration with chickpea grain yield under drought stress (Taleei and shaabani, 2016a; Saxena et al. 1993). Farooq et al. (2016) said that the main reasons terminal drought-mediated loss of grain yield are rate of net photosynthesis reduction, decrease in grain and damage to seed development. In grain legumes as chickpea, stay green longer can be a sufficient potential for increased duration pod fillingmediated more yield (Rong-hua et al. 2006). A collection include 211 chickpea accessions was stablished in three years, shoot biomas and grain yield showed a highly heritability than other such as phenological traits. It said as well as top yielding accessions had a flowering time up to 45-50 days following sowing seeds, among the set in two years, but this time was 40 DAS for thired (Krishnamurthy et al. 2010). Our observations, with respected to the results, suggested any association, whether drought or irrigated condition, has not between flowering time and yield (Tables 4, 5, and 6). In the evaluating 
of canopy temperature depression effect on yield of chickpea under terminal drought, was initiated at 62 days after sowing, nearly podding time, it was a significant positive correlation between those (Purushothaman et al. 2015). Podding time has, in the present work, also similar positive association with yield, though at non-stress condition (Table 5). A drought-induced decline percent of seed yield was estimated 49-54, 27-40, 45-69 for late ripening, anthesis, and reproductive stage in chickpea, respectively (Samarah et al. 2009; Mafakheri et al. 2010; Nayyar et al. 2006). It seems that plant phenology behaviorinfluenced outcomes of dissection trials could been penetrated by many factors as genetic, environment, season, temperature, moment beginning, severity and duration of water deficit, soil profile and many of others (Taleei and shaabani, 2016b). Chapman et al. (2000a) pronounced stress-mediated temporal and spatial unpredictability could make a gap between environments where these factors already limit growth and yield of chickpea and multi-field experiments. The mismatch may be suffice for converse genetic improvement, as in the many of studies in effect with drought stress, the early flowering genotypes are favorite, whereas have a few yield in most desirable environments (Chapman et al. 2000b). The results of modeling to known the pattern of water deficit and temperature determined environment as a dominant source of variation for Australian chickpea yield and/or production (Lake et al. 2016). Krishnamurthy et al. (2010) confirmed that the highly drought sensitive accessions (ICC 3776, ICC 8058 and ICC 7184) have a greater canopy than rest. Total dry matter, in the present work has a great correlation with seed yield. This trait known as a follower of growth and development events, such as water absorption of root, canopy, or shoot mass, efficiency of radiations, etc, as shown in the tables 4,5 , and $6, \mathrm{r}$ (coefficient of correlation) was equal to 0.404 , 0.977 , and 0.941 for irrigated, drought, and mean values of those conditions, respectively. 100-seed weight was a unique character that did affected not by drought (Table 2). Varshney et al. (2014) reported that chickpea's 100-seed weight has least in effect with water scarcity for several years and locations; also its heritability was high that's why supposed selection based on this trait could be positive advancement in more and stable yield breeding programs under terminal drought stress. Cluster analysis on traits-obtained data placed the genotypes 321 and 322 in a same group; these genotypes, moreover, were located with the genotype 10 at a distinc group based on tolerance indices (Figuers 1 and 2). Although phenological characters did not show any difference between the three more tolerant genotypes, diversity of those in yield and number of seed was considerable (Table 2 ). The grain yield has greatest correlation with seed number and total dry matter for stress and non-stress condition, respectively. Factor analysis also proposed the total dry matter, yield, number of seed, and flowering time as mian traits in the case of conditions, in order of importance (Table 8). According to the above discussion, genotype 321 known as the most tolerant one followed by genotypes 10 and 322, respectively. The results showed that the intended genotypes had short time to flowering and podding stages, as applying of stress was at $50 \%$ flowering time of overall genotypes.

\section{Acknowledgments}

We acknowledge The Iranian Pulse Project Exellence for supporting this work.

\section{References}

[1] M. Bänziger, G. O. Edmeades and H. R. Lafitte, "Selection for Drought Tolerance Increases Maize Yields across a Range of Nitrogen Levels", Crop Science., vol. 39, no. 4, (1999), pp. 1035-1040.

[2] S. C. Chapman, M. Cooper, G. L. Hammer and D. G. Butler, "Genotype by Environment Interactions Affecting Grain Sorghum. II. Frequencies of Different Seasonal Patterns of Drought Stress Are Related to Location Effects on Hybrid Yields", Crop and Pasture Science, vol. 51, no. 2, (2000a), pp. 209-222.

[3] S. C. Chapman, G. L. Hammer, D. G. Butler, and M. Cooper, "Genotype by Environment Interactions Affecting Grain Sorghum. III, Temporal Sequences and Spatial Patterns in the Target Population of Environments", Crop and Pasture Science, vol. 51, no. 2, (2000b), pp. 223-234. 
[4] M. Farooq, N. Gogoi, S. Barthakur, B. Baroowa, N. Bharadwaj, S.S. Alghamdi and K. H. M. Siddique, "Drought Stress in Grain Legumes during Reproduction and Grain Filling", Journal of Agronomy and Crop Science, (2016).

[5] M. Farooq, A. Wahid, N. Kobayashi, D. Fujita and S. M. A. Basra, "Plant Drought Stress: Effects, Mechanisms and Management", Sustainable Agriculture, Springer Netherlands, (2009). pp. 153-188

[6] C. Fernandez, "Effective Selection Criteria for Assessing Plant Stress Tolerance", In Proceedings of the International Symposium on Adaptation of Vegetables and other Food Crops in Temperature and Water Stress, (1992), pp. 257-270.

[7] R. A. Fischer, D. Rees, K. D. Sayre, Z. M. Lu, A. G. Condon and A. L. Saavedra, "Wheat yield progress associated with higher stomatal conductance and photosynthetic rate, and cooler canopies", Crop science, vol. 38 , no. 6, (1998), pp. 1467-1475.

[8] S. Fukai and M. Cooper, "Development of Drought-Resistant Cultivars Using Physiomorphological Traits in Rice", Field Crops Research, vol. 40, no. 2, (1995), pp. 67-86.

[9] A. E. Hall, "Is Dehydration Tolerance Relevant to Genotypic Difference in Leaf Senescence and Crop Adaption to Dry Environments?", Current Topics in Plant Physiology, USA, (1993).

[10] L. Jusheng, "Comparison of Evaluating Methods for Agronomic Drought Resistance in Crops", Acta Agriculturae Boreali-Occidentalis Sinica, vol. 3, (1998).

[11] Krishnamurthy, J. Kashiwagi, P. M. Gaur, H. D. Upadhyaya and V. Vadez, "Sources of Tolerance to Terminal Drought in The Chickpea (Cicer Arietinum L.) Minicore Germplasm”, Field Crops Research, vol. 119, no. 2, (2010), pp. 322-330.

[12] Lake and V. O. Sadras, "Screening Chickpea for Adaptation to Water Stress: Associations between Yield and Crop Growth Rate", European Journal of Agronomy, vol. 81, (2016), pp. 86-91.

[13] Lake, K. Chenu and V. O. Sadras, "Patterns of Water Stress and Temperature for Australian Chickpea Production", Crop and Pasture Science, vol. 67, no. 2, (2016), pp. 204-215.

[14] R. H. Li, P. G. Guo, B. Michael, G. Stefania and C. Salvatore, "Evaluation of Chlorophyll Content and Fluorescence Parameters as Indicators of Drought Tolerance in Barley", Agricultural Sciences in China, vol. 5, no. 10, (2006), pp. 751-757.

[15] M. M. Ludlow and R. C. Muchow, "A Critical Evaluation of Traits for Improving Crop Yields in WaterLimited Environments", Advances in Aagronomy, vol. 43, (1990), pp.107-153.

[16] Mafakheri, A. Siosemardeh, B. Bahramnejad, P. C. Struik and Y. Sohrabi, "Effect of Drought Stress on Yield, Proline and Chlorophyll Contents in Three Chickpea Cultivars", Australian Journal of Crop Science, vol. 4, no. 8, (2010), p. 580.

[17] H. Nayyar, S. Kaur, S. Singh and H. D. Upadhyaya, "Differential Sensitivity of Desi (Small-Seeded) and Kabuli (Large-Seeded) Chickpea Genotypes to Water Stress During Seed Filling: Effects on Accumulation of Seed Reserves and Yield”, Journal of the Science of Food and Agriculture, vol. 86, no. 13, (2006), pp. 2076-2082.

[18] Purushothaman, M. Thudi, L. Krishnamurthy, H. D. Upadhyaya, J. Kashiwagi, C. L. L. Gowda and R. K. Varshney, "Association of Mid-Reproductive Stage Canopy Temperature Depression with the Molecular Markers and Grain Yields of Chickpea (Cicer Arietinum L.) Germplasm under Terminal Drought", Field Crops Research, vol. 174, (2015), pp. 1-11.

[19] P. Ramirez-Vallejo and J. D. Kelly, "Traits Related to Drought Resistance in Common Bean", Euphytica, vol. 99, no. 2, (1998), pp.127-136.

[20] Rosielle and J. Hamblin, "Theoretical Aspects of Selection for Yield in Stress and Non-Stress Environment", Crop Science", vol. 21, no. 6, (1981), pp. 943-946.

[21] H. Sabaghpour, A. A. Mahmodi, A. Saeed, M. Kamel and R. S. Malhotra, "Study on Chickpea Drought Tolerance Lines under Dryland Condition of Iran”, Indian Journal of Crop Science, vol. 1, no. 1-2, (2006), pp. 70-73.

[22] V. Sadras and M. F. Dreccer, "Adaptation of Wheat, Barley, Canola, Field Pea and Chickpea to the Thermal Environments of Australia", Crop and Pasture Science, vol. 66, no. 11, (2015), pp. 1137-1150.

[23] O. Sadras, L. Lake, Y. Li, E. A. Farquharson and T. Sutton, "Phenotypic Plasticity and Its Genetic Regulation for Yield, Nitrogen Fixation and $\Delta 13 \mathrm{c}$ in Chickpea Crops under Varying Water Regimes", Journal of Experimental Botany, vol. 67, no. 14, (2016), pp. 4339-4351.

[24] N. H. Samarah, N. Haddad, and A. M. Alqudah, "Yield Potential Evaluation in Chickpea Genotypes under Late Terminal Drought in Relation to the Length of Reproductive Stage", Italian Journal of Agronomy, vol. 4, no, 3, (2009), pp. 111-117.

[25] N. P. Saxena, L. Krishnamurthy and C. Johansen, "Registration of a Drought-Resistant Chickpea Germplasm", Crop Science, vol. 33, (1993), p. 1424.

[26] K. A. Schneider, R. Rosales-Serna, F. Ibarra-Perez, B. Cazares-Enriquez, J. A. Acosta-Gallegos, P. Ramirez-Vallejo, N. Wassimi and J. D. Kelly, "Improving Common Bean Performance under Drought Stress", Crop Science, vol. 37, no. 1, (1997), pp. 43-50.

[27] V. K. Singh, A. W. Khan, D. Jaganathan, M. Thudi, M. Roorkiwal, H. Takagi, V. Garg, V. Kumar, A. Chitikineni, P. M. Gaur and T. Sutton, "QTL-Seq for Rapid Identification of Candidate Genes for 100-Seed Weight and Root/Total Plant Dry Weight Ratio under Rainfed Conditions in Chickpea”, Plant Biotechnology Journal, (2016). 
[28] A. Taleei and J. shaabani, "Yield potential Analysis of Kabuli Chickpea Genotypes in Water Stress Conditios", Advanced Science and Technology Letters, vol. 142, (2016), pp. 1-8

[29] A. Taleei and J. shaabani, "Yield potential Analysis of Desi Chickpea Genotypes in Water Stress Conditios" Advanced Science and Technology Letters, vol. 142, (2016), pp. 9-16.

[30] N. C. Turner, G. C. Wright and K. H. M. Siddique, "Adaptation of Grain Legumes (Pulses) to WaterLimited Environments", Advances in Agronomy, vol. 71, (2001), pp. 193-231.

[31] R. K. Varshney, M. Thudi, S. N. Nayak, P. M. Gaur, J. Kashiwagi, L. Krishnamurthy, D. Jaganathan, J. Koppolu, A. Bohra, S. Tripathi and A. Rathore, "Genetic Dissection of Drought Tolerance in Chickpea (Cicer Arietinum L.). Theoretical and Applied Genetics", vol. 127, no. 2, (2014), pp. 445-462.

[32] J. W. White and J. A. Castillo, "Evaluation of Diverse Shoot Genotypes on Selected Root Genotypes of Common Bean under Soil Water Deficits", Crop Science, vol. 32, no. 3, (1992), pp. 762-765.

[33] L. Wiegand and A. J. Richardson, "Use of Spectral Vegetation Indices To Infer Leaf Area, Evapotranspiration And Yield: I. Rationale", Agronomy Journal, vol. 82, no. 3, (1990), pp. 623-629.

[34] F. XiangWen, N. C. Turner, Y. GuiJun, L. FengMin and K. H. M. Siddique, "Flower Numbers, Pod Production, Pollen Viability, and Pistil Function are Reduced and Flower and Pod Abortion Increased in Chickpea (Cicer Arietinum L.) Under Terminal Drought", Journal of Experimental Botany, vol. 61, no. 2, (2010), pp. 335-345. 
International Journal of Bio-Science and Bio-Technology Vol.9, No.1 (2017) 